\title{
Design and Optimization of High-Speed Resonant Cavity Enhanced Schottky Photodiodes
}

\author{
Mutlu Gökkavas, Bora M. Onat, Student Member, IEEE, Ekmel Özbay, E. P. Ata, J. Xu, E. Towe, \\ and M. Selim Ünlü, Senior Member, IEEE
}

\begin{abstract}
Resonant cavity enhanced (RCE) photodiodes (PD's) are promising candidates for applications in optical communications and interconnects where high-speed high-efficiency photodetection is desirable. In RCE structures, the electrical properties of the photodetector remain mostly unchanged; however, the presence of the microcavity causes wavelength selectivity accompanied by a drastic increase of the optical field at the resonant wavelengths. The enhanced optical field allows to maintain a high efficiency for faster transit-time limited PD's with thinner absorption regions. The combination of an $\mathrm{RCE}$ detection scheme with Schottky PD's allows for the fabrication of high-performance photodetectors with relatively simple material structures and fabrication processes. In top-illuminated RCE Schottky PD's, a semitransparent Schottky contact can also serve as the top reflector of the resonant cavity. We present theoretical and experimental results on spectral and high-speed properties of GaAs-AlAs-InGaAs RCE Schottky PD's designed for 900-nm wavelength.
\end{abstract}

Index Terms-High-speed optoelectronics, photodetectors, photodiodes, resonant cavity, Schottky diodes.

\section{INTRODUCTION}

$\mathbf{S}$ CHOTTKY photodiodes (PD's) are very attractive for high-speed photodetection because of their simple material structure. Their relatively simple fabrication process enables easy integration with III-V discrete devices and integrated circuits. Furthermore, since the junction is at the metal-semiconductor interface, diffusion of photogenerated carriers and parasitic series resistance in a Schottky PD is less when compared to pin PD's, resulting in superior high-speed performance. Schottky PD's with 3-dB bandwidths well above $100 \mathrm{GHz}$ have been reported [1]. However, these devices had low quantum efficiencies as a result of thin absorption layers necessary for high-speed operation. The quantum efficiency $(\eta)$ of a vertically illuminated (photons and charged carriers travel along the same axis) pin PD for $\alpha d \ll 1$ is given by [2]

$$
\eta \approx(1-R) \alpha d
$$

where $\alpha$ is the absorption coefficient, $d$ is the thickness of the

Manuscript received April 10, 1998; revised October 9, 1998. This work was supported in part by the Office of Naval Research under Grant N0001496-1-0652, by the National Science Foundation under Grant INT-96-01770, and by the Turkish Scientific and Technical Research Council under Project EEEAG-156.

M. Gökkavas, B. M. Onat, and M. S. Ünlü are with the Center for Photonics Research and the Department of Electrical and Computer Engineering, Boston University, Boston, MA 02215 USA.

E. Özbay and E. P. Ata are with the Department of Physics, Bilkent University, Bilkent, Ankara 06533, Turkey.

J. Xu and E. Towe are with the Department of Electrical Engineering, University of Virginia, Charlottesville, VA 22903 USA.

Publisher Item Identifier S 0018-9197(99)00852-0. absorber, and $R$ is the reflectivity of the PD. On the other hand, in the transit-time limit, the bandwidth of the device is inversely proportional to $d$

$$
f_{3-\mathrm{dB}} \approx 0.45 \mathrm{v} / \mathrm{d}
$$

where $v$ is the saturation velocity of the photogenerated carriers. Hence thinner absorption regions are employed for higher bandwidths. Conventional high-speed PD's, therefore, suffer a low quantum efficiency.

Equations (1) and (2) also show that the bandwidth-efficiency product (BWE) is independent of the design parameter $d$ and depends only on material parameters $\alpha$ and $v$. Although typical III-V compound semiconductors have very large $\alpha$ values resulting in high BWE at visible wavelengths, at optical communication wavelengths (1.3 and $1.55 \mu \mathrm{m}$ and recently $850 \mathrm{~nm}) \alpha$ and thus BWE remain limited. For example, for GaAs detectors operating around $850 \mathrm{~nm}$, this product is less than $30 \mathrm{GHz}$. To overcome the BWE limitation of single-pass photodetectors, different photodetection schemes can be employed. Edge-illuminated waveguide detectors that separate the optical and electrical axes have achieved a $110-\mathrm{GHz}$ bandwidth and quantum efficiency of 0.5 at $1.55 \mu \mathrm{m}$ [3]. The disadvantages of edgeilluminated detectors are complex fabrication and integration along with difficult light coupling. Resonant cavity enhanced (RCE) photodetection is another method that has been successfully demonstrated over the past few years [4], [5]. In RCE, the much preferred vertical illumination capability is not sacrificed; instead, the quantum efficiency of the device is improved by placing a thin absorbing layer inside a Fabry-Perot microcavity. By using RCE, vertically illuminated devices with both high speed and high $\eta$ can be realized. Electrical isolation of RCE devices is typically accomplished by mesa etch, a disadvantage toward the integration of these devices into planar fabrication technologies. However, doped GaAs can be made semi-insulating by proton bombardment [1], and, using this technique, the RCE Schottky PD's reported in this paper, as well as other RCE structures, can also be fabricated without a deep mesa etch, which should allow their integration with planar structures.

Schottky PD's are particularly attractive for RCE detection. Besides standard advantages such as ease of fabrication, lower contact resistance, and lower diffusion current which also apply to conventional Schottky PD's, there are several advantages, both electrical and optical, unique to the RCE design. In RCE devices, from an optical standpoint, a shorter cavity is preferred [4]. In the case of RCE pin PD's, this causes 
the $\mathrm{P}^{+}$and $\mathrm{N}^{+}$contacts to be thin layers with a relatively high sheet resistance adversely affecting the temporal response. Furthermore, RCE pin PD's usually employ distributed Bragg reflectors (DBR's) as a high-reflectivity top mirror. In contrast, when a top-illuminated RCE scheme is employed, a thin semitransparent Schottky contact [1], [6] readily eliminates the lateral access resistance problem, allows for a shorter cavity, and increases the air-semiconductor interface reflectivity [7] without the need for a top DBR. The cavity resonance can be tuned to the desired wavelength by recessing the top surface prior to fabrication [8], [9], which is a significant advantage of these devices. While the bandwidth-efficiency improvement of RCE detection has been fully exploited for pin [10] and avalanche PD's [11], there have been only a few reports on RCE Schottky PD's [7], [12], [13]. The first RCE Schottky PD was reported by Chin et al. [12]. They used a $1500-\AA ̊$ high-reflectivity Al Schottky contact and a 16-layer InAlAs-InGaAlAs DBR to form the detector microcavity. The light was coupled into the cavity from the InP substrate side (bottom illumination) through the DBR and was absorbed by a $4750-\AA ̊$ InGaAs absorbing region. These devices showed $50 \%$ enhancement in photocurrent over conventional control devices. Tzeng et al. reported the first high-speed results from RCE Schottky PD's [13]. Their device was a top-illuminated GaAs Schottky PD with a $1-\mu \mathrm{m}$ GaAs absorber and an eightlayer GaAs-AlAs DBR at the bottom. In their model, they assumed the 100- $\AA$-thick semitransparent Au Schottky contact layer to be fully transparent for top illumination and treated the air-semiconductor interface as the top mirror of the detector cavity. For a $25-\mu$ m-diameter device, they achieved a $9-\mathrm{GHz}$ $3-\mathrm{dB}$ bandwidth. They also measured a $30 \%$ improvement in the responsivity over a single-pass device. In both these attempts, the potential of the RCE concept was not fully exploited, mostly due to relatively thick absorption regions.

To date, AlGaAs-GaAs-InGaAs has been the most commonly studied material system in RCE research [4] because of the well-established growth processes. The close lattice match in the ternary $\mathrm{AlGaAs}$ and the large refractive index difference [14] between the two binary endpoints AlAs and GaAs allow the growth of high-quality high-reflectivity DBR's with a reasonable number of layers. We fabricated RCE Schottky PD's operating in two different wavelength regions (850 and 900 $\mathrm{nm}$ ) that are dictated by the optical properties of the materials used. When the photon energy of the incident light is less than the GaAs bandgap energy, GaAs can be used as a lowloss DBR material, greatly simplifying the growth process. While a thin InGaAs absorber allows the device spectrum to be extended to wavelengths above the GaAs band edge [15], the maximum attainable pseudomorphic InGaAs thickness limits the wavelength coverage to $860 \mathrm{~nm}$ to $1 \mu \mathrm{m}$. We use this material system mainly to demonstrate the RCE Schottky PD concept around $900 \mathrm{~nm}$ [7], [16]. With the advent of GaAs vertical-cavity surface-emitting lasers (VCSEL's) [17], the wavelength range around $850 \mathrm{~nm}$ has recently emerged as a strong candidate for short-distance high-bit-rate parallel fiber optic communication systems. We believe that highspeed RCE Schottky PD's can perfectly complement VCSEL arrays and concentrate our further efforts on the fabrication of

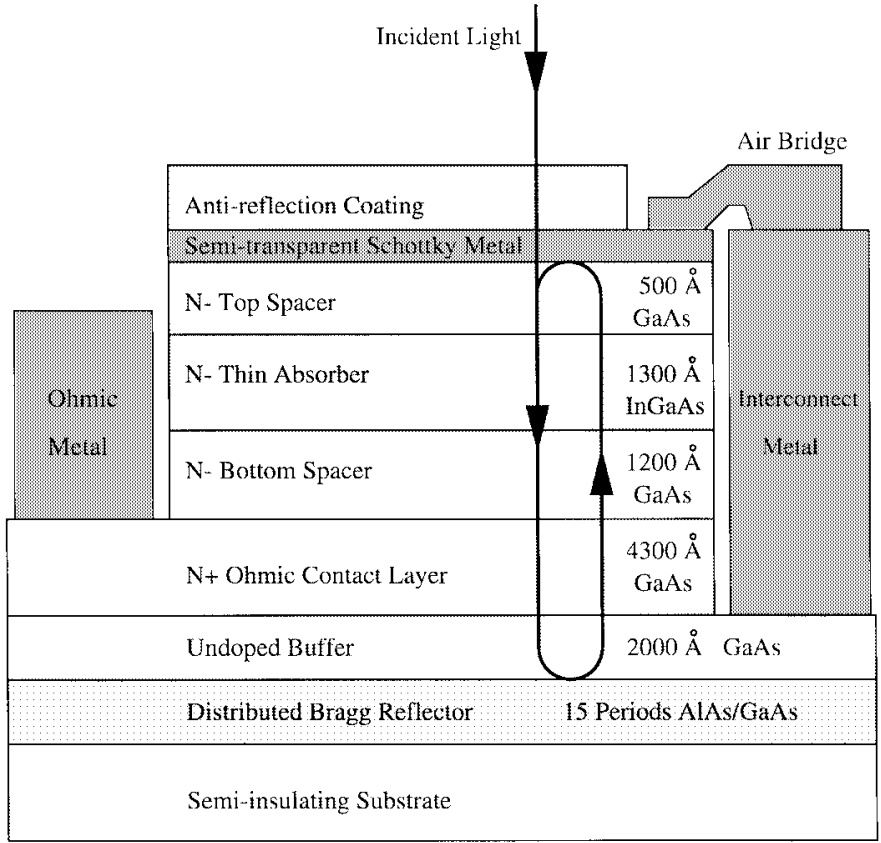

Fig. 1. Conceptual cross section of a top-illuminated RCE Schottky PD and the corresponding layer structure for $900-\mathrm{nm}$ operation.

devices operating in the 820-850-nm range. In this wavelength regime, GaAs is used as the absorber material; however it is too lossy to be used in the DBR structure. Instead, a low-loss DBR can be formed by alternating $\mathrm{Al}_{x} \mathrm{Ga}_{1-x} \mathrm{As}-\mathrm{AlAs}$ layers [18].

In this paper, we first address the design strategy for a RCE Schottky PD structure, considering both high-speed and high-efficiency characteristics. Also, a detailed analysis of the expected performance for the 900-nm devices and a discussion of various design parameters are included. A brief description of device fabrication and experimental results on both spectral photoresponse and high-speed properties of the 900-nm RCE Schottky PD's are presented (data on the performance of devices for around 850-nm operation is presented elsewhere [18]). Finally, an interpretation of experimental results and comparisons with the theoretical predictions are included.

\section{DESIGN OF RCE SCHOTTKY PD}

Fig. 1 shows the cross section of a conceptual topilluminated RCE Schottky PD. The resonant microcavity is formed between the semitransparent Schottky contact and a low-loss DBR. An $\mathrm{N}^{-}$depletion region, an $\mathrm{N}^{+}$ohmic contact layer, and an undoped buffer constitute the different electrical regions of the PD in the cavity. The depletion region consists of a top spacer, a bottom spacer, and a thin absorption layer sandwiched between these layers. All layers but the absorber inside the cavity have a larger bandgap than the incident light energy. Hence, the cavity exhibits a very low loss with absorption occurring only in the thin absorber. An antireflection (AR) coating may also be deposited on top of the Schottky metal to optimize the detector frontsurface reflectivity, thereby increasing the maximum attainable efficiency. 
The quantum efficiency for a simplified RCE PD structure with lossless mirrors is given by Kishino et al. [5] as

$$
\begin{aligned}
\eta= & \left\{\frac{\left(1+R_{2} e^{-\alpha d}\right)}{1-\sqrt{R_{1} R_{2}} e^{-\alpha d} \cos \left(2 \beta L+\psi_{1}+\psi_{2}\right)+R_{1} R_{2} e^{-2 \alpha d}}\right\} \\
& \times\left(1-R_{1}\right)\left(1-e^{-\alpha d}\right)
\end{aligned}
$$

where $\alpha$ and $d$ are the absorption coefficient and thickness for the thin absorber, $R_{1}$ and $R_{2}$ are the top and bottom mirror reflectivities, respectively, $\beta$ is the propagation constant, $L$ is the cavity length, and $\psi_{1}$ and $\psi_{2}$ are the phase shifts introduced by the top and bottom mirrors, respectively. On the righthand side of (3), the term in the braces represents the power enhancement factor. When $R_{2}=0$, this term becomes unity, and (3) gives $\eta$ for a conventional detector. Hence, the power enhancement factor represents the ratio of quantum efficiencies of RCE and conventional detectors with the same $\alpha d$. In the case of an absorptive mirror, such as the semitransparent metallic top mirror of RCE Schottky PD's, (3) is no longer valid. Nevertheless, the qualitative dependency of $\eta$ on many design parameters can still be deduced from (3). The peak wavelength $\left(\lambda_{p}\right)$ is a function of $L, \psi_{1}$, and $\psi_{2}$. The maximumattainable $\eta$, on the other hand, depends on $R_{1}, R_{2}$, and the normalized absorption coefficient $\alpha d$. For a low-loss cavity (high $R_{1}$ and $R_{2}$ and low $\alpha d$ ), at the resonant wavelengths, the optical field amplitude inside the microcavity is increased, thus $\eta$ is drastically enhanced.

The important limitations to the response speed of an RCE Schottky PD are generally transit time of the carriers (transittime limit) across the depletion region and charging and discharging times of the inherent and parasitic capacitances [2], [4]. Other limiting factors are charge trapping at heterojunctions and diffusion from undepleted regions. These can be eliminated, respectively, by grading the absorbing region heterojunctions and by placing the absorbing region between nonabsorbing spacers in the depletion region. The transit-time response of an RCE PD is much shorter than an equal quantum efficiency conventional PD, because the latter has to employ a much thicker absorption region to accomplish the same efficiency. It is also predicted that an RCE pin PD has a $35 \%$ larger bandwidth than a conventional pin PD with the same depletion layer thickness [19], when the absorption layer is placed in an optimum position inside the depletion region, thereby equalizing the arrival times of the faster electrons and slower holes. These arguments are equally valid for Schottky PD's, and the transit time of the designed structure with a $0.3-\mu \mathrm{m}$ depletion layer is expected to be $3 \mathrm{ps}$. The capacitancelimited response time of the devices can be decreased below that of the transit time by reducing the device area.

In the case of a top-illuminated design, to obtain a high $Q$ cavity, the bottom DBR reflectivity should be as high as possible [4], [5]. However, the number of layers in the DBR cannot be indefinitely increased because of the practical difficulties encountered in the molecular beam epitaxy (MBE) growth of such structures. Therefore, one has to make a compromise between the DBR reflectivity and the structural quality of the DBR. Reasonably good DBR quality and a high bottom reflectivity $\left(R_{2}=0.97\right)$ can be obtained for
15 periods of alternating AlAs-GaAs layers as employed in our devices. Since there are both forward- and backwardpropagating fields in the cavity, the magnitude of the total optical electric field $(E(z))$ exhibits a half-wavelength $(\lambda / 2 n)$ period standing wave pattern. Equation (3) neglects the effect of the standing wave pattern. The power enhancement factor is more accurately expressed in terms of the standing wave as

$$
\frac{\eta_{\mathrm{RCE}}}{\eta_{\text {conventional }}}=\frac{\int\left|E_{\mathrm{RCE}}(z)\right|^{2} d z}{\int\left|E_{\text {conventional }}(z)\right|^{2} d z}
$$

where both integrations have to be carried out between the respective absorber boundaries. In (4), the subscripts have been used in the same manner as the previous definition of the enhancement factor. The relative positioning of the thin absorber with respect to the standing wave pattern is hence critical for very thin absorbing layers and is an important design consideration [4]. To obtain the maximum enhancement possible, the absorber has to coincide with a maximum of the standing wave pattern. However, an accurate positioning of a thin absorber with respect to the standing wave pattern is difficult, since an error of a few percent in the layer thicknesses during the growth process is common. To eliminate this risk, a $\lambda / 2 n$-thick absorber is used, hence exactly one period of the standing wave pattern coincides with the absorber. Another important design consideration is the actual value of $\alpha$. If $\alpha$ is too small, a thick absorption layer is required, degrading the high-speed properties of the device by increasing the carrier transit time. On the other hand, large values of $\alpha$ requires very thin absorbers in order to keep the total cavity loss low. However, this emphasizes the complications associated with the standing wave effect (SWE). The respective values of $\alpha$ for InGaAs around $900 \mathrm{~nm}$ and for GaAs around $850 \mathrm{~nm}$ are expected to be $1.3 \times 10^{4} \mathrm{~cm}^{-1}$ [15] and $9 \times 10^{3} \mathrm{~cm}^{-1}$ [14], yielding a moderate normalized absorption coefficient $(\alpha d \sim 0.15)$ for $d \sim \lambda / 2 n$.

\section{ANALYSIS OF RCE SCHOTTKY PD'S}

The quantitative dependency of the photoresponse on various design parameters is investigated using a scattering matrix ( $S$ matrix) approach [13]. In the simulations, the complex refractive indices [14], [15], [20] of the materials were used to calculate the $S$ matrices for each layer in Fig. 1, which were cascaded to calculate the forward- and backward-propagating optical fields in the entire multilayer structure. The field distribution was used to optimize the final device structure by calculating the dependency of quantities such as frontsurface reflectivity, total transmission through the device, the absorption in each layer, and the device quantum efficiency on various design parameters. Below, we report sample simulation results.

In Fig. 2, the first three periods of the standing wave pattern for the optimized $900-\mathrm{nm}$ structure is plotted. For clarity, the forward- and backward-propagating field amplitudes are also shown. This simulation was performed for a 200- $\AA$ thick $\mathrm{Au}$ Schottky layer and a 1050 - $\AA$-thick $\mathrm{Si}_{3} \mathrm{~N}_{4}$ dielectric AR coating. The horizontal axis in the figure represents the position along the cavity, and the boundaries of various layers 


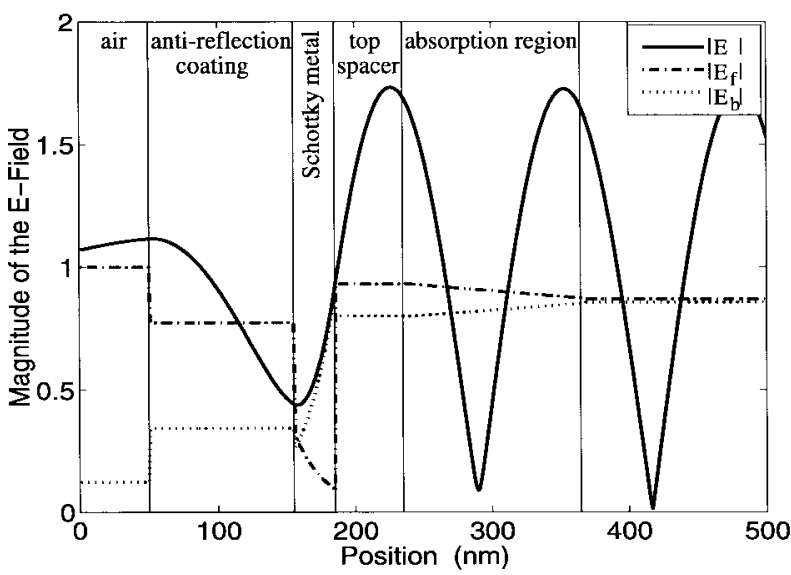

Fig. 2. Simulation showing the first few periods of the standing wave pattern inside the RCE Schottky PD. The wavelength is $900 \mathrm{~nm}$, the bottom mirror is a 15-period DBR, the top mirror is the 200- $\AA$-thick Au Schottky metal, and the AR coating is a 1050 - $\AA$-thick $\mathrm{Si}_{3} \mathrm{~N}_{4}$ layer.

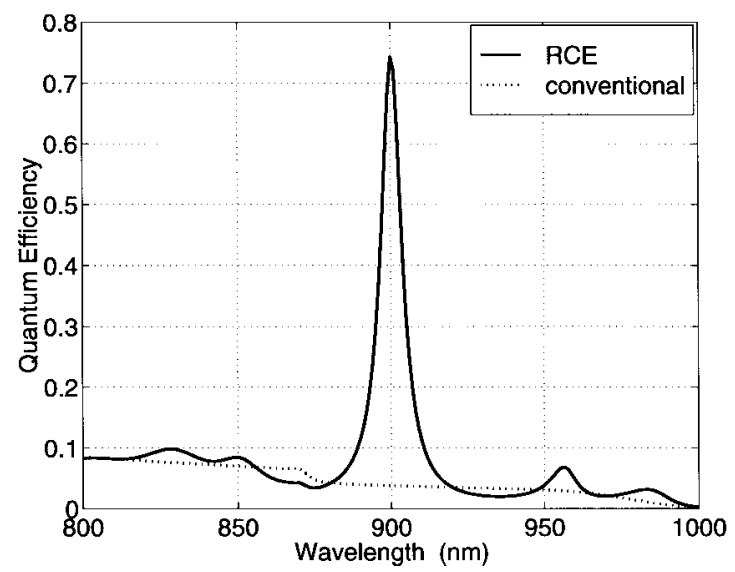

Fig. 3. Theoretical comparison of the calculated quantum efficiency (solid curve) for the RCE Schottky PD structure in Fig. 1 and that of a conventional Schottky PD (dashed curve) with the same structure except the DBR.

are shown by vertical lines. The standing wave amplitude of $\sim 1.75$ corresponds to a power enhancement factor in excess of 10. Also note that the backward-propagating field amplitude is much larger than the forward-propagating field amplitude in the metallic layer. Hence, most of the power absorbed in the metal is due to the enhanced field inside the cavity. This suggests that a higher $\eta$ will be accompanied by an increased absorption in the metal. It is calculated that $20 \%$ of the incident power will be absorbed in the metal for the optimized structure, significantly larger than that for a single pass.

Fig. 3 shows the calculated quantum efficiency spectra of the optimized device. The expected peak quantum efficiency $\left(\eta_{p}\right)$ at $\lambda_{p}=900 \mathrm{~nm}$ is $\sim 0.7$. The full-width at half maximum (FWHM) of the photoresponse is expected to be $7 \mathrm{~nm}$, which together with the 70-nm free spectral range yields a finesse value of 10. For comparison, the quantum efficiency attainable from an identical structure without the DBR is also shown. With the RCE scheme, a more then tenfold improvement in $\eta$ is observed.

For fixed values of bottom mirror reflectivity and absorber thickness, $\eta$ will be maximized when the front-surface (detec-

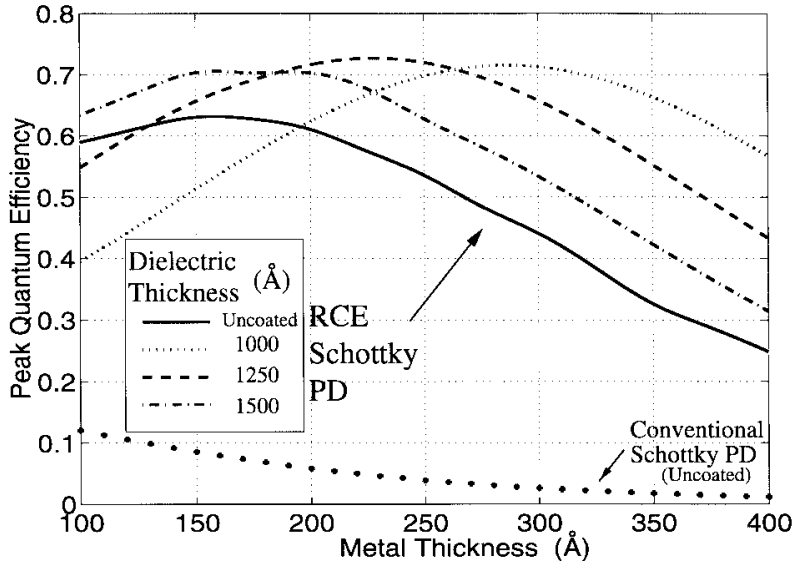

Fig. 4. Expected peak quantum efficiency of the structure in Fig. 1 versus Schottky metal thickness for different values of the dielectric coating thickness. The calculated quantum efficiency of a single-pass conventional Schottky $\mathrm{PD}$ with a $0.3-\mu \mathrm{m}$ GaAs absorber is also shown.

tor) reflectivity for the incident light (see Fig. 1) is minimized [4], [5]. To achieve this condition, a dielectric AR coating on top of the Schottky contact is used. By varying the thicknesses of the Au Schottky metallization $\left(t_{m}\right)$ and the $\mathrm{Si}_{3} \mathrm{~N}_{4}$ dielectric coating $\left(t_{d}\right), \eta$ can be significantly enhanced over a wide range of metal thickness values. This is particularly desirable in applications where minimizing the series contact resistance is important. In Fig. $4, \eta_{p}$ versus $t_{m}$ for different values of $t_{d}$ is plotted. For the RCE Schottky PD structure in Fig. 1, calculated $\eta_{p}$ is well over 0.5 for $t_{m}<400 \AA$, whereas $\eta$ for a conventional Schottky PD gradually decreases by increasing $t_{m}$.

An important design consideration is the robustness of the device performance against deviations from the optimized layer structure during the growth and fabrication processes. The device performance is mostly sensitive to the bottom mirror quality, a high-reflectivity DBR being an essential element of the RCE concept. Variations on the order of a few percent in the layer thicknesses during MBE process are common, causing the resonance wavelength and/or the DBR center wavelength to shift. Our simulations predict that, for the $\lambda / 2 n$ absorber at hand, a peak quantum efficiency larger than 0.6 is attainable for a $30-\mathrm{nm}$ wavelength range around the DBR center wavelength by adjusting the top spacer, Schottky metal, and AR coating thicknesses. Since all these parameters are post-growth alterable, the device can be tuned to a desired wavelength in a $30-\mathrm{nm}$ range without a significant quantum efficiency sacrifice.

\section{EXPERIMENTAL RESULTS}

The epitaxial layers were grown by solid-source MBE on top of semi-insulating GaAs substrate. The optimized layer thicknesses for the 900-nm RCE Schottky PD are shown in Fig. 1. The structure consists of 15 periods of AlAs-GaAs quarter-wave layers $\left(R_{2}=0.97\right)$ followed by a $0.2-\mu \mathrm{m}$ undoped buffer, a $0.43-\mu \mathrm{m} \mathrm{N}^{+}$GaAs ohmic contact layer, and a $0.3-\mu \mathrm{m} \mathrm{N} \mathrm{N}^{-}$depletion region. The depletion region incorporates a $1300-\AA \operatorname{In}_{0.08} \mathrm{Ga}_{0.92} \mathrm{As}$ absorber sandwiched between a $500-\AA \AA$ top spacer and a $1200-\AA$ bottom spacer 


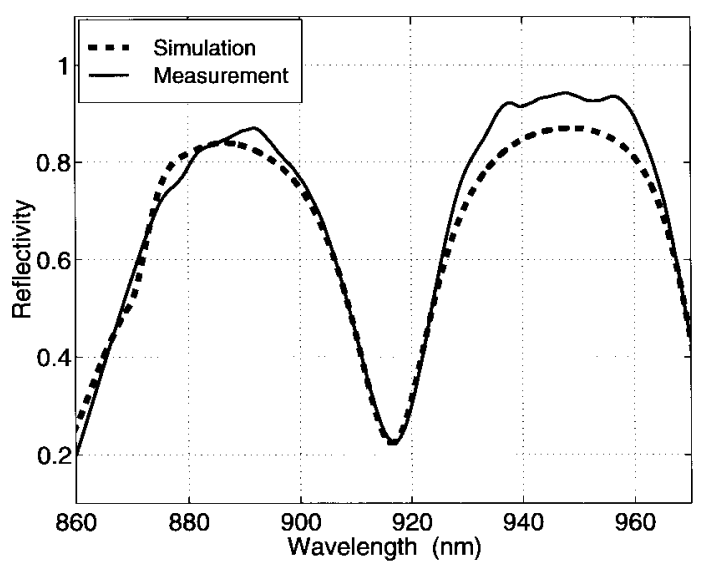

Fig. 5. Measured (solid) and calculated (dashed) front-surface reflectivities for the MBE wafer prior to fabrication.

chosen to equalize hole and electron transit times. The total depletion region thickness was chosen as $0.3 \mu \mathrm{m}$ to ensure transit-time limited operation for the smaller area devices. The absorber heterojunctions were linearly graded to minimize electron and hole trapping at the interfaces. Before device fabrication, reflectivity of the grown MBE wafers were measured using a spectrophotometer. Fig. 5 shows this spectral measurement and a theoretical fit to the data, demonstrating a good agreement, hence the validity of the simulation method used. The reflectivity spectra of the DBR was observed to be shifted toward longer wavelengths (possibly due to variations during MBE growth). Also, from the fit to the data, it is predicted that $\alpha$ is approximately a factor of two higher than the expected value. However, for an RCE PD, $\eta$ is not a strong function of $\alpha$, and this higher value of $\alpha$ does not correspond to a significant increase in maximum attainable $\eta$. To obtain the maximum value of $\eta$ for a given value of $\alpha$, however, other cavity parameters have to be optimized accordingly. Since the cavity was optimized for a lower $\alpha$ value, the higher than expected $\alpha$ should in fact result in a lower than expected $\eta$ value.

The epitaxial wafers were fabricated using a monolithic microwave compatible process. Fig. 1 shows a cross section of the fabricated devices. First, $\mathrm{N}^{-}$layers were recess etched and $\mathrm{Au}-\mathrm{Ge}-\mathrm{Ni}$ ohmic contacts to the $\mathrm{N}^{+}$layer were formed by a self-aligned lift-off and a rapid thermal anneal. This was followed by the deposition of the 200- $\AA$ semitransparent Schottky contact. All areas except the active device area were then etched away and a Ti-Au interconnect metal was evaporated to form coplanar waveguide (CPW) transmission line patterns on top of the semi-insulating substrate. Next, a 2000-Å-thick $\mathrm{Si}_{3} \mathrm{~N}_{4}$ AR coating was deposited and patterned. This layer also passivates and protects the surface and serves as the metal-insulator-metal bias capacitor dielectric. To minimize the parasitic capacitance of the devices, fabrication was completed by a $1.5-\mu \mathrm{m}$-thick Au airbridge that connects the Schottky metal to the center conductor of the CPW [21].

The fabricated devices had different mesa sizes varying from $150 \mu \times 150 \mu \mathrm{m}$ for spectral measurements to $7 \mu \times 5 \mu \mathrm{m}$ for high-speed measurements. Devices with intermediate mesa sizes were also fabricated for investigating the bandwidth-area

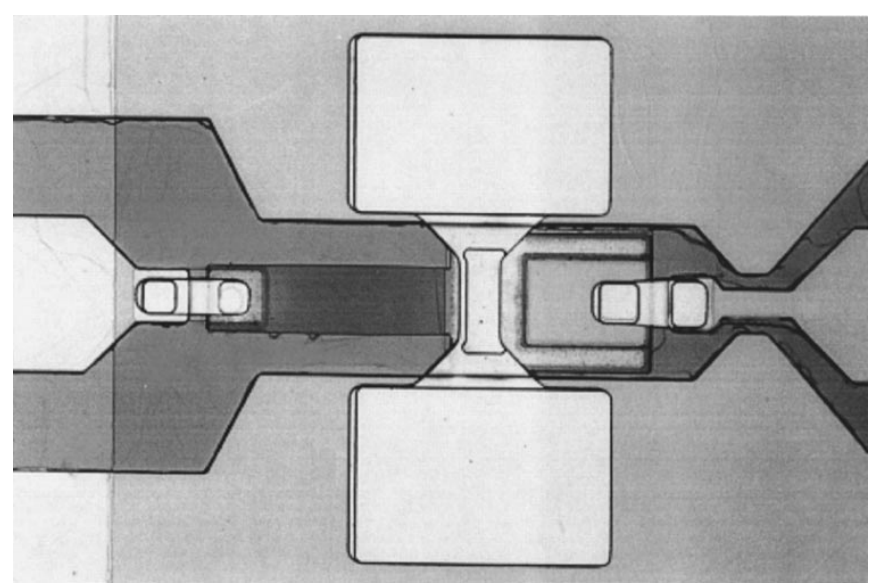

Fig. 6. Photomicrograph of a $150 \mu \times 150 \mu \mathrm{m}$ device.

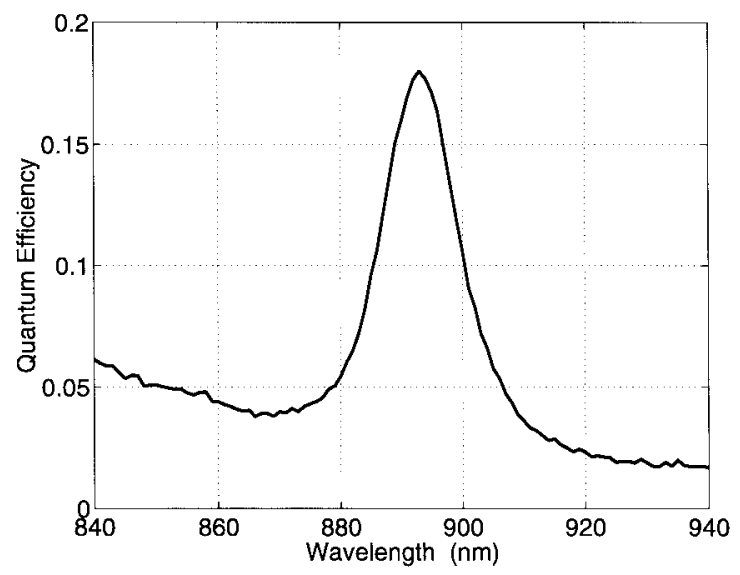

Fig. 7. Measured spectral response of RCE Schottky PD's. The peak quantum efficiency $\eta_{p}$ is $18 \%$ at $895 \mathrm{~nm}$, FWHM is $15 \mathrm{~nm}$, and the enhancement factor is $\sim 6$.

dependence. Fig. 6 shows a photomicrograph of a $150 \mu \times 150$ $\mu \mathrm{m}$ detector. The Schottky diodes exhibit breakdown voltages larger than $12 \mathrm{~V}$. The dark current of a $150 \mu \times 150 \mu \mathrm{m}$ device at $-1-\mathrm{V}$ bias was $30 \mathrm{nA}$. Using forward $I-V$ characterization, the Schottky barrier height was measured to be $0.83 \mathrm{eV}$.

Fig. 7 shows the measured photoresponse spectra of the devices. The peak quantum efficiency was measured to be $18 \%$ at $895 \mathrm{~nm}$ whereas the expected value was above $70 \%$. Nevertheless, when compared with a single-pass device, the enhancement factor is 6 and the FWHM is $15 \mathrm{~nm}$. To further investigate the dependency of the peak quantum efficiency on the metal thickness, a reflectivity experiment was performed. Without fabricating the devices, Au layers with different thicknesses $(100,150$, and $200 \AA)$ were evaporated on an epitaxial wafer sample. Then, $2000-\AA \mathrm{Si}_{3} \mathrm{~N}_{4} \mathrm{AR}$ coating was deposited on the sample and was etched in $200-\AA$ steps. After each etch, the front-surface reflectivity was measured. There was good agreement between the theory and the data for both the resonance wavelengths and the FWHM of the resonance dip. However, the resonant front-surface reflectivity was measured to be higher than expected. It was also noted that the minimum front-surface reflectivity for the fabricated devices was attained using a thinner Au layer (100 $\AA$ ) than 


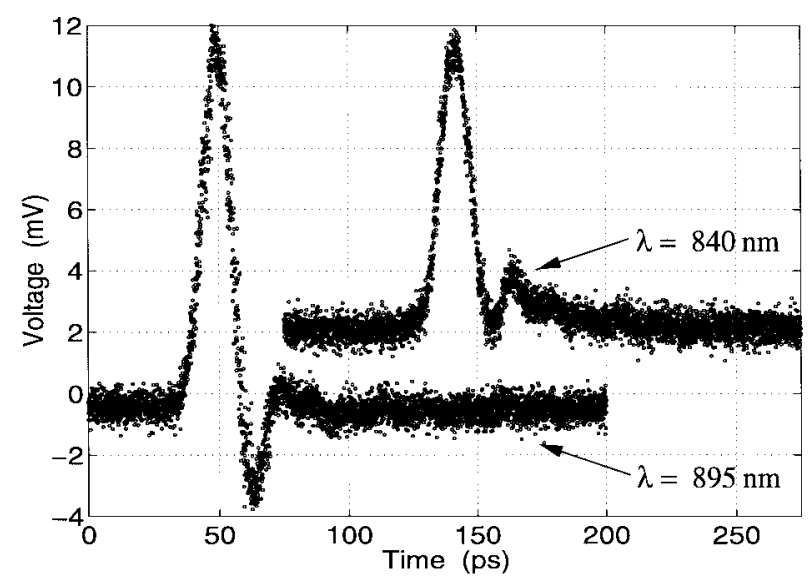

Fig. 8. Pulse response of $10 \times 10 \mu \mathrm{m}$ RCE Schottky PD with an $\mathrm{In}_{0.08} \mathrm{Ga}_{0.92} \mathrm{As}$ absorber. Measured FWHM is $10 \mathrm{ps}$ at resonance (895 $\mathrm{nm})$, and $13 \mathrm{ps}$ at off-resonance $(840 \mathrm{~nm})$. The 10-ps FWHM measured by the scope is estimated to correspond to a 4.1-ps FWHM impulse response.

the value predicted by the simulations (150 ̊). Using atomic force microscopy (AFM) measurements, significant roughness (30 $\AA \mathrm{rms}$ for $100-\AA$ mean thickness) on the surface of the Au metallization was observed. Two possible sources of the high discrepancy between the expected and measured $\eta$ values are: 1) scattering and depolarization losses [22] due to this surface roughness, which were not taken into account during simulations that assume planar boundaries between layers, and 2) possible misfit dislocations in the lattice-mismatched absorber. For a similar In concentration, the critical thickness of InGaAs is reported to be $>2000$ $\AA$ [23]. Also, visual inspection of the wafer and measured low dark current indicate good crystalline quality. Hence, we attribute the lower than expected $\eta$ to the Au surface roughness. Incorporating the effect of surface roughness in the simulation model and improving the surface quality of the metallization process is currently being investigated. When the roughness problem of the Schottky contact is eliminated, it should be possible to achieve the $70 \% \eta$ predicted by the simulations. On similar devices operating around $850-\mathrm{nm}$ wavelength, where a more controlled experiment in terms of the Schottky metal thickness and the absorption coefficient of the thin absorber was performed, $\eta$ values as high as $50 \%$ and a 25-GHz BWE were demonstrated [18].

High-speed measurements were performed using a picosecond mode-locked $\mathrm{Ti}$ : sapphire laser. The devices were illuminated using a single-mode fiber on a microwave probe station and the response at zero bias was observed with a $50-\mathrm{GHz}$ sampling oscilloscope. For the wavelength dependence of the high-speed response, a $10 \mu \mathrm{m} \times 10 \mu \mathrm{m}$ device was studied. Fig. 8 shows the pulse response obtained from the same device under two different excitation wavelengths. At 895 $\mathrm{nm}$, the incident light is absorbed only in the thin InGaAs absorber (see Fig. 1) with the exception of the Schottky metal, the rest of the cavity is nearly lossless, and the device functions as an RCE Schottky PD. On the other hand, at $840 \mathrm{~nm}$, both GaAs and InGaAs are absorptive. At this wavelength, the DBR reflectivity is less than 0.2 , and only a small fraction of the incident light reaches this low-reflectivity
DBR considering the $0.93-\mu \mathrm{m}$ cavity length. Thus, the device functions essentially as a conventional single-pass Schottky $\mathrm{PD}$, and the pulse response obtained is very close to what would have been observed from a conventional Schottky PD with a $0.3-\mu \mathrm{m}$ depletion region. The FWHM's of the pulse responses as measured on the scope were $10 \mathrm{ps}$ at $895 \mathrm{~nm}$ and $13 \mathrm{ps}$ at $840 \mathrm{~nm}$. The measured rise time, which is a characteristic of the measurement setup, was 9 ps at both wavelengths. However, the fall time was significantly shorter at the resonant wavelength than at the single-pass wavelength (6 ps at $895 \mathrm{~nm}$ versus $10 \mathrm{ps}$ at $840 \mathrm{~nm}$ ). Since the capacitance limit on the bandwidth is independent of wavelength, the dependence of the fall time on the wavelength of excitation clearly demonstrates the transit-time-limited nature of both measurements. When the device is functioning as an RCE Schottky PD, photogeneration of carriers occurs only in the InGaAs absorber, at the optimum position where the transit times for holes and electrons are equal, whereas at offresonance the transit-time-limited response is dominated by the slower carriers and holes. When the as-measured FWHM pulsewidths are compared, the improvement with RCE is only $30 \%$. However, the pulse responses observed on the scope correspond to the total system bandwidth and are limited by the speed of the sampling scope. The detector pulsewidth $\left(\tau_{\text {detector }}\right)$ can be approximated as [24]

$$
\tau_{\text {detector }}=\sqrt{\tau_{\text {total }}^{2}-\tau_{\text {scope }}^{2}-\tau_{\text {optical }}^{2}} .
$$

This is very accurate for Gaussian pulses and a good approximation in our case. Considering a 9-ps FWHM pulsewidth for the scope [25] and a 1.2-ps laser pulsewidth, the estimated detector impulse width is $4.1 \mathrm{ps}$ at $895 \mathrm{~nm}$ and $9.3 \mathrm{ps}$ at $840 \mathrm{~nm}$, respectively [16]. This is a conservative estimate since the microwave components in the signal path and laser timing jitter also contribute to the measured pulsewidth. The corresponding 3-dB bandwidth is in excess of $100 \mathrm{GHz}$ at 895 $\mathrm{nm}$ and $47 \mathrm{GHz}$ at $840 \mathrm{~nm}$, demonstrating a more than twofold improvement in bandwidth with the RCE detection scheme as predicted by simulations [19]. Together with the nearly threefold increase in $\eta$ (see Fig. 7), the bandwidth-efficiency product of the devices is improved by a factor of 6 at resonance $(895 \mathrm{~nm})$ versus at off-resonance $(840 \mathrm{~nm})$.

To further verify the transit-time-limited operation of these devices, the high-speed response of various area devices were measured. The devices with mesa sizes smaller than $20 \mu \times 30$ $\mu \mathrm{m}$ were observed not to be capacitance limited, demonstrating impulse responses with $\sim 10$ ps FWHM. The capacitance of the $10 \mu \times 10 \mu \mathrm{m}$ device is calculated as $\sim 40 \mathrm{fF}$, corresponding to a capacitance-limited bandwidth of more than $200 \mathrm{GHz}$. The theoretical expected transit-time limited FWHM at $895 \mathrm{~nm}$ is 3 ps in good agreement with the experiment.

The fast Fourier transforms (FFT's) of the as-measured impulse responses (Fig. 8) when the RCE Schottky PD is at resonance $(895 \mathrm{~nm})$ and when it is at off-resonance $(840 \mathrm{~nm})$ are shown in Fig. 9. To accurately represent the frequency response for the displayed frequency range, two different data sets (4096 samples covering 200 ps and 1 ns total span, respectively) for each pulse were used. The reason for the 


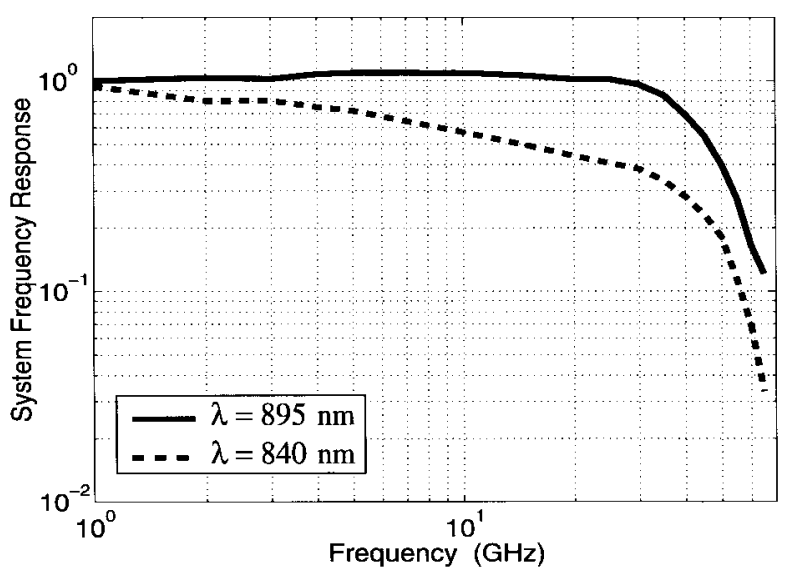

Fig. 9. Frequency response of the system obtained by fast Fourier transforming the data in Fig. 8. There is a gradual drop at lower frequencies for the $840-\mathrm{nm}$ response.

gradual drop in the lower frequency portion of the 840$\mathrm{nm}$ response in Fig. 9 is the long tail observable in the corresponding pulse response in Fig. 8. Since a substantial portion of the incident light at $840 \mathrm{~nm}$ is absorbed in the undepleted $\mathrm{N}^{+}$region and beyond, carrier diffusion from these regions causes a long tail in pulse response. In comparison, since there is no significant photocarrier generation outside the thin InGaAs absorber at $895 \mathrm{~nm}$, this tail due to diffusion is absent in Fig. 8, and the corresponding frequency response in Fig. 9 is flat. Hence, with the RCE detection scheme, further improvement is observed in the frequency domain than is revealed by the simple pulsewidth comparisons in the time domain.

\section{CONCLUSIONS}

In this paper, the concept of combining the respective advantages of resonant cavity enhancement and Schottky PD's to achieve high-performance, efficient high-speed photodetection has been investigated. The design strategy has been demonstrated for an (Al, In)GaAs-based top-illuminated RCE Schottky PD operating around $900 \mathrm{~nm}$. Dependency of highspeed and spectral performance on various design parameters have been discussed and can be summarized as follows.

1) Quantum efficiency is maximized when front-surface reflectivity for incident light is minimized. To minimize the front-surface reflectivity, a dielectric layer can be used, and by adjusting the metal and dielectric thicknesses, a high quantum efficiency can be maintained over a large range of these parameters.

2) The reflectivity of the bottom DBR should be the maximum allowed within the budget of the growth process.

3) As the optical field inside the cavity increases, so does the absorption in the semitransparent Schottky contact. Hence, a unity quantum efficiency cannot be achieved. However, quantum efficiency values in the $70 \%-80 \%$ range can be achieved for practical values of the absorber thickness and the DBR reflectivity.

4) The value of $\alpha d$ should be on the order of 0.1 to observe practical benefit of RCE. This condition is easily satisfied for GaAs between $800-860 \mathrm{~nm}$ and for $\mathrm{In}_{x \sim 0.1} \mathrm{Ga}_{1-x}$ As around $900 \mathrm{~nm}$.

5) For very thin absorbers, SWE can drastically degrade the efficiency as a result of small variations in the layer thicknesses during the growth procedure. The design can be made immune to SWE by choosing $d=\lambda / 2 n$.

6) High-speed design is independent from spectral efficiency design. Depletion region thickness can be chosen with respect to the area of the device to assure transittime-limited operation. The thin absorber position within the depletion region can be exploited to equalize carrier transit times. Absorber boundaries can be graded to avoid carrier trapping at the heterojunctions.

Following these guidelines, we have designed and fabricated RCE Schottky PD's in the (Al, In)GaAs material system with a semitransparent Au Schottky contact and top mirror for operation at $900 \mathrm{~nm}$. The measured high-speed response agrees with theoretical predictions of expected improvement by the RCE detection scheme. The 10-ps FWHM pulses on the scope are estimated to represent 4.1-ps FWHM impulse response, corresponding to a $3-\mathrm{dB}$ bandwidth of over $100 \mathrm{GHz}$. When the Au surface smoothness is improved, the optimized structure is expected to yield a bandwidth-efficiency product larger than $70 \mathrm{GHz}$.

\section{REFERENCES}

[1] E. Özbay, K. D. Li, and D. M. Bloom, "2.0 ps, $150 \mathrm{GHz}$ GaAs monolithic photodiode and all-electronic sampler," IEEE Photon. Technol. Lett., vol. 3, pp. 570-572, June 1991.

[2] J. E. Bowers and C. A. Burrus, "Ultrawide-band long-wavelength p-i-n photodetectors," J. Lightwave Technol., vol. LT-5, pp. 1339-1350, Oct. 1987.

[3] K. Kato, A. Kozen, Y. Muramoto, Y. Itaya, T. Nagatsuma, and M. Yaita, "110-GHz, 50\%-efficiency mushroom-mesa waveguide p-i-n photodiode for $1.55-\mu \mathrm{m}$ wavelength," IEEE Photon. Technol. Lett., vol. 6, pp. 719-721, June 1994.

[4] M. S. Ünlü, and S. Strite, "Resonant cavity enhanced photonic devices," J. Appl. Phys., vol. 78, no. 2, pp. 607-639, 1995.

[5] K. Kishino, M. S. Ünlü, J. Chyi, J. Reed, L. Arsenault, and H. Morkoç, "Resonant cavity-enhanced (RCE) photodetectors," IEEE J. Quantum Electron., vol. 27, pp. 2025-2034, Aug. 1991.

[6] R.-H. Yuang, J.-I. Chyi, Y.-J. Chan, W. Lin, and Y.-K. Tu, "Highresponsivity InGaAs MSM photodetectors with semitransparent Schottky contacts," IEEE Photon. Technol. Lett., vol. 7, pp. 1333-1335, Nov. 1995 .

[7] E. Özbay, M. S. Islam, B. M. Onat, M. Gökkavas, O. Aytür, G. Tuttle, E. Towe, R. H. Henderson, and M. S. Ünlü, "Fabrication of high-speed resonant cavity enhanced Schottky photodiodes," IEEE Photon. Technol. Lett., vol. 9, pp. 672-674, May 1997.

[8] S. S. Murtaza, I. H. Tan, J. E. Bowers, E. H. Lu, K. A. Anselm, M. R. Islam, R. V. Chelakara, R. D. Dupuis, B. G. Streetman, and J. C. Campbell, "High-finesse resonant-cavity photodetectors with an adjustable resonance frequency," J. Lightwave Technol., vol. 14, pp. 1081-1089, June 1996.

[9] M. S. Ünlü, S. Taşıran, A. L. Demirel, and H. Morkoç, "Low cross-talk monolithic wavelength demultiplexing switch arrays," in Proc. 1992 Bilkent Int. Conf. on Lightwave Communication, Ankara, Turkey, 1992.

[10] C. C. Barron, C. J. Mahon, B. J. Thibeault, G. Wang, W. Jiang, L. A. Coldren, and J. E. Bowers, "Resonant-cavity-enhanced pin photodetector with $17 \mathrm{GHz}$ bandwidth-efficiency product," Electron. Lett., vol. 30, no. 21, pp. 1796-1797, 1994.

[11] H. Nie, K. A. Anselm, C. Hu, S. S. Murtaza, B. G. Streetman, and J. C. Campbell, "High-speed resonant-cavity separate absorption and multiplication avalanche photodiodes with $130 \mathrm{GHz}$ gain-bandwidth product," Appl. Phys. Lett., vol. 70, no. 2, pp. 161-163, 1997.

[12] A. Chin and T. Y. Chang, "Enhancement of quantum efficiency in thin photodiodes through absorptive resonance," J. Lightwave Technol., vol. 9, pp. 321-328, Mar. 1991. 
[13] Y. Tzeng, S. S. Li, and P. Ho, “A GaAs Schottky-barrier photodiode with high quantum efficiency-bandwidth product by using a multilayer reflector," IEEE Trans. Electron Devices, vol. 40, pp. 348-352, Feb. 1993.

[14] D. E. Aspnes, S. M. Kelso, R. A. Logan, and R. Bhat, "Optical properties of $\mathrm{Al}_{x} \mathrm{Ga}_{1-x} \mathrm{As}, "$ J. Appl. Phys., vol. 60, no. 2, pp. 754-767, 1986.

[15] S. A. Alterovitz, "Dielectric functions and refractive index of $\mathrm{In}_{x} \mathrm{Ga}_{1-x} \mathrm{As}, "$ in Optical and Electro-Optic Properties of InGaAs. London, U.K.: INSPEC, 1993.

[16] B. M. Onat, M. Gökkavas, E. Özbay, E. P. Ata, E. Towe, and M. S. Ünlü, "100 GHz resonant cavity enhanced Schottky photodiodes," IEEE Photon. Technol. Lett., vol. 10, pp. 707-709, May 1998.

[17] W. W. Chow, K. D. Choquette, M. H. Crawford, K. L. Lear, and G. R. Hadley, "Design, fabrication, and performance of infrared and visible vertical-cavity surface-emitting lasers," IEEE J. Quantum Electron., vol. 33, pp. $1810-1824$, Oct. 1997.

[18] M. S. Ünlü, M. Gökkavas, B. Onat, E. Ata, E. Özbay, R. P. Mirin, K. J. Knopp, K. A. Bertness, and D. H. Christensen, "High bandwidthefficiency resonant cavity enhanced Schottky photodiodes for 800-850 nm operation wavelength," Appl. Phys. Lett., vol. 72, no. 21, pp. $2727-2729,1998$

[19] M. S. Ünlü, B. M. Onat, and Y. Leblebici, "Transient simulation of heterojunction photodiodes-Part II: Analysis of resonant cavity enhanced photodetectors," J. Lightwave Technol., vol. 13, pp. 406-415, Mar. 1995.

[20] P. B. Johnson and R. W. Christy, "Optical constants of the noble metals," Phys. Rev. B, vol. 6, no. 12, pp. 4370-4379, 1972.

[21] E. Özbay, D. M. Bloom, D. H. Chow, J. N. Schulman, "1.7-ps, microwave, integrated-circuit-compatible InAs/AlSb resonant tunneling diodes," IEEE Electron Device Lett., vol. 14, pp. 400-402, Aug. 1993.

[22] E. Bahar and R. D. Kubik, "Computations of the Mueller matrix elements for scattering from layered structures with rough surfaces, with applications to optical detection," Appl. Opt., vol. 36, no. 13, pp. 2947-2955, 1997.

[23] C. Carter-Coman, R. Bicknell-Tassius, A. S. Brown, and N. M. Jokerst, "Analysis of $\mathrm{In}_{0.07} \mathrm{Ga}_{0.93}$ As layers on GaAs compliant substrates by double crystal x-ray diffraction," Appl. Phys. Lett., vol. 70, no. 13, pp. 1754-1756, 1997.

[24] K. J. Weingarten, M. J. W. Rodwell, and D. M. Bloom, "Picosecond optical sampling of GaAs integrated circuits," IEEE J. Quantum Electron. vol. 24, pp. 198-220, Feb. 1988.

[25] K. Rush, S. Draving, and J. Kerley, "Characterizing high-speed oscilloscopes," IEEE Spectrum, vol. 27, pp. 38-39, Sept. 1990

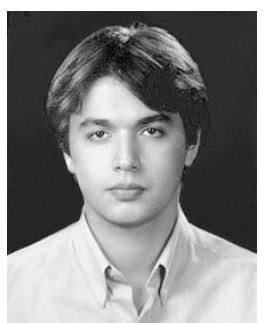

Mutlu Gökkavas was born in Nazilli, Turkey, on May 15, 1972. He received the B.S. and M.S degrees in electrical engineering from Bilkent University, Ankara, Turkey, in 1994 and 1996, respectively. He is currently working toward the Ph.D. degree at Boston University, Boston, MA

He joined the Electrical and Computer Engineering Department, Boston University, as a Research Assistant in 1996. His research interests include design, fabrication, characterization, and modeling of semiconductor optoelectronic devices, and modeling and characterization of high-speed measurements and setups.

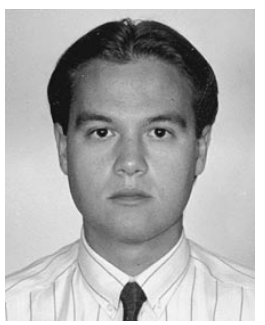

Bora M. Onat (S'92) was born in Melrose, MA, in 1971. He received the B.S. degree in electrical engineering from Istanbul Technical University, Istanbul, Turkey, in 1992 and the M.S. and Ph.D. degrees in electrical engineering from Boston University, Boston, MA, in 1995 and 1998, respectively.

$\mathrm{He}$ is currently employed by Lucent Technologies, Breinigsville, PA.

Dr. Onat has received a Graduate Assistantship in Area of National Need (GAANN) fellowship, the EG\&G Photonics Fellowship Award, and the Melles Griot Award in Photonics Research in 1996, and he has won the SPIE student paper competition Runner Up Award and Honorable Mention Award in 1997 and 1994, respectively.

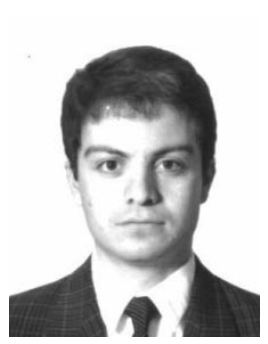

Ekmel Özbay was born in Ankara, Turkey, on March 25, 1966. He received the B.S. degree in electrical engineering from the Middle East Technical University, Ankara, Turkey, in 1983 and the M.S. and Ph.D. degrees in electrical engineering from Stanford University, Standford, CA, in 1989 and 1992, respectively. During his thesis work, he focused on high-speed resonant tunneling and optoelectronic devices.

From 1992 to 1994, he worked as a Scientist in DOE Ames National Laboratory, Iowa State University, Ames, in the area of photonic bandgap materials. He joined the Faculty of the Physics Department, Bilkent University, Ankara, Turkey, in December 1994, where he is currently an Associate Professor. His research at Bilkent involves photonic crystals, silicon micromachining, and high-speed optoelectronics. He has authored or co-authored more than 70 articles in scientific journals, conference proceedings, and books.

Dr. Özbay is the 1997 recipient of the Adolph Lomb Medal of the Optical Society of America.

E. P. Ata, photograph and biography not available at the time of publication.

J. Xu, photograph and biography not available at the time of publication.

E. Towe, photograph and biography not available at the time of publication.

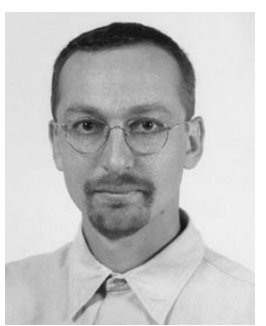

M. Selim Ünlü (S'90-M'92-SM'95) was born in Sinop, Turkey, in 1964. He received the B.S. degree in electrical engineering from Middle East Technical University, Ankara, Turkey, in 1986 and the M.S.E.E. and Ph.D. degrees in electrical engineering from the University of Illinois, Urbana-Champaign, in 1988 and 1992, respectively.

In 1992, he joined the Department of Electrical and Computer Engineering, Boston University, Boston, MA, as an Assistant Professor, and he has been an Associate Professor since 1998. His research interests include design, fabrication, characterization, and modeling of semiconductor optoelectronic devices, near-field and picosecond spectroscopy, integrated optical biosensors, and optical pumping for production of hyperpolarized Xe for MRI.

Dr. Ünlü served as the Chair of IEEE Laser and Electro-Optics Society, Boston Chapter, during 1994-1995, winning the LEOS Chapter-of-the-Year Award. He was also awarded the United Nations TOKTEN award in 1995 and 1996, and both the National Science Foundation CAREER Award and the Office of Naval Research Young Investigator Award in 1996. 